\title{
Limits to Electron Beam Emittance from Stochastic Coulomb Interactions
}

\author{
Christopher Coleman-Smith, ${ }^{*}$ Howard A. Padmore, and Weishi Wan ${ }^{\dagger}$ \\ Advanced Light Source, Lawrence Berkeley National Laboratory, Berkeley, CA 94720
}

(Dated: April 30, 2009)

\begin{abstract}
Dense electron beams can now be generated on an ultrafast timescale using laser driven photocathodes and these are used for a range of applications from ultrafast electron defraction to free electron lasers. Here we determine a lower bound to the emittance of an electron beam limited by fundamental stochastic Coulomb interactions.
\end{abstract}

PACS numbers: 29.27.Bd, 41.75.Ht, 41.85.Ct 
The advances in diverse fields such as electron microscopy and the free electron laser drive demand for high brightness electron sources[1]. Apart from the intrinsic properties of the cathode material, the Coulomb interaction among emitted electrons is the other major factor that limits the brightness of the final beam. In fact, these two factors are intertwined such that the optimal source for a given system is a compromise between them. The study of Coulomb interactions among electrons has a long history $[2,3]$ and has become progressively more active due to increasing brightness requirements [4]. In order to achieve higher brightness than the state of the art, a more detailed understanding of this effect is needed.

This paper is an attempt to extend understanding of Coulomb interactions among electrons emitted from a cathode. Since the stochastic parts of the coulomb interaction are characterised by short-length scale phenomena, collisions, we adopt a bottom-up approach and utilize N-Body methods from astrophysics [5]. This is in marked contrast to the spatially discretized particle-in-cell (PIC) methods [6, 7] commonly used in electron transport codes. The main advantage of this method is that no spatial grid is needed, which ensures that all length-scales of the Coulomb interaction are included up to the limits of numerical precision. Yet, for direct summation of the coulomb interaction over the beam, the scaling law of $\mathrm{O}\left(\mathrm{N}^{2}\right)$ for the computation time poses a limit on the number of electrons that can be realistically simulated (roughly $10^{4}$ for a PC). The tree-code algorithm developed by Barnes and Hut [8] is an elegant solution. Instead of direct summation, the force on each electron is approximated by lumping distant electrons, which fall inside a certain solid angle, into one macro particle. As a result, the scaling law for the computation time becomes $\mathrm{O}(\mathrm{N} \log \mathrm{N})$ and increases the upper limit of the number of electrons to the order of $10^{6}$ on a small computer.

The Coulomb interactions among emitted electrons manifest themselves in two ways. One is the correlated expansion of the beam due to the mean field, which is sometimes referred to as the space charge effect. The other is the uncorrelated or stochastic scattering, which has different names in different fields. In this paper, we shall refer to it as the stochastic Coulomb interaction. The main difference between the two effects is that the space charge effect conserves the phase space volume whereas the stochastic Coulomb interaction leads to an increase in phase space volume. The space charge effect generates various correlations in the phase space which may result in the degradation of the brightness of the beam. It has been demonstrated that the linear part of the correlated motion can be removed [9] 
and in principle the nonlinear parts may be removed as well [10]. The stochastic Coulomb interactions, on the other hand, result in an irreversible increase of the phase space volume and concomitant degradation of brightness. Hence it is of crucial importance that a detailed knowledge of this effect be obtained.

In our simulation, two steps were taken to isolate the stochastic Coulomb interactions from the space charge effect. First, the geometric shape and distribution of the bunch are chosen such that the nonlinear correlations are absent. It is well known that the electrostatic field in a homogeneous ellipsoid is linear in all directions [11]. Hence only linear correlations are present in the phase space, which is true for all time as long as the external field is also linear in space [12]. As a result, a homogeneous ellipsoid is used throughout this paper unless stated otherwise. Secondly, the linear correlations are removed through evaluating the determinant of the $6 \times 6$ second-order moment matrix of the phase space variables, which is the $6 \mathrm{D}$ emittance of the electron bunch. Specifically, the phase space variables are $x, p_{x}$ $=P_{x} / m_{e} c, y, p_{y}=P_{y} / m_{e} c, z$ and $p_{z}=P_{z} / m_{e} c$, where $m_{e}$ is the rest mass of the electron and $c$ is the speed of light. The rms $6 \mathrm{D}$ emittance is defined as

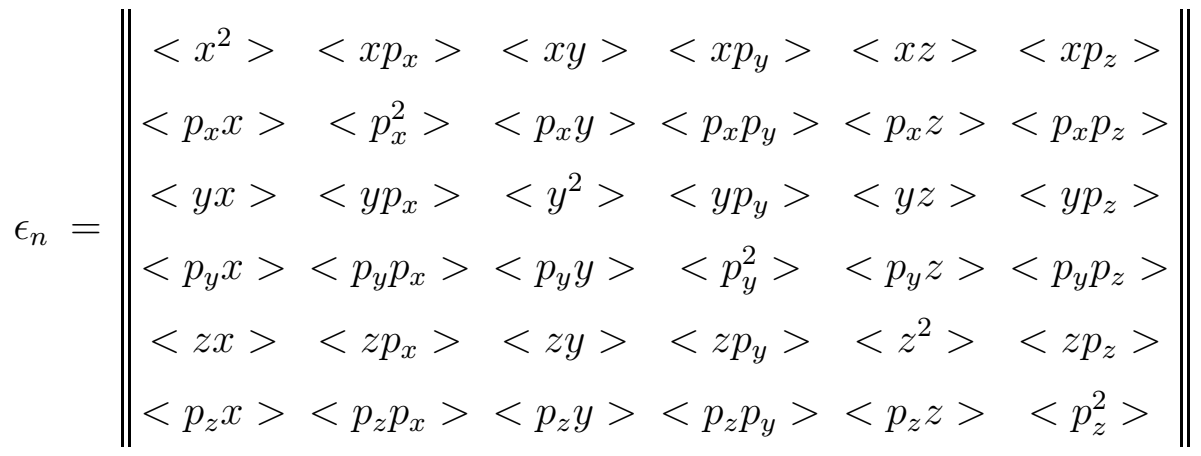

$$
\begin{aligned}
& \left\|\begin{array}{||cccccc}
<x^{2}> & <x \gamma \beta_{x}> & <x y> & <x \gamma \beta_{y}> & <x z> & <x \gamma \beta_{z}> \\
<\gamma \beta_{x} x> & <\gamma^{2} \beta_{x}^{2}> & <\gamma \beta_{x} y & <\gamma \beta_{x} \gamma \beta_{y}> & <\gamma \beta_{x} z & <\gamma \beta_{x} \gamma \beta_{z}> \\
<y x> & <y \gamma \beta_{x}> & <y^{2}> & <y \gamma \beta_{y}> & <y z> & <y \gamma \beta_{z}> \\
<\gamma \beta_{y} x & <\gamma \beta_{y} p_{x}> & <\gamma \beta_{y} y & <\gamma^{2} \beta_{y}^{2}> & <\gamma \beta_{y} z & <\gamma \beta_{y} \gamma \beta_{z}> \\
<z x> & <z \gamma \beta_{x}> & <z y> & <z \gamma \beta_{y}> & <z^{2}> & <z \gamma \beta_{z}> \\
<\gamma \beta_{z} x> & <\gamma \beta_{z} \gamma \beta_{x}> & <\gamma \beta_{z} y & <\gamma \beta_{z} \gamma \beta_{y}> & <\gamma \beta_{z} z & <\gamma^{2} \beta_{z}^{2}>
\end{array}\right\|,
\end{aligned}
$$

where $\gamma$ is the relativistic factor which is the ratio of the total energy and the rest energy of a particle and $\beta_{i}=v_{i} / c$ for $i=x, y, z$. For a homogeneous ellipsoidal bunch, all higher-order moments, hence all nonlinear correlations, vanish. Furthermore, the $r m s$ 6D emittance $\epsilon_{n}$ 
is an invariant of motion in the mean field, which entails that all linear correlations are removed and any change in $\epsilon_{n}$ is due to the stochastic Coulomb interactions.

Due to the rapid expansion of the electron bunch in the vacuum, from both the space charge effect and an external accelerating field, the stochastic Coulomb interactions diminishes quickly. As it becomes clear below, the time range the stochastic Coulomb interactions are important is typically on the order of 100 ps. As a result, the energy of the electrons remains low for typical accelerating fields and a non-relativistic approximation is sufficient. Hence the rms 6D emittance becomes

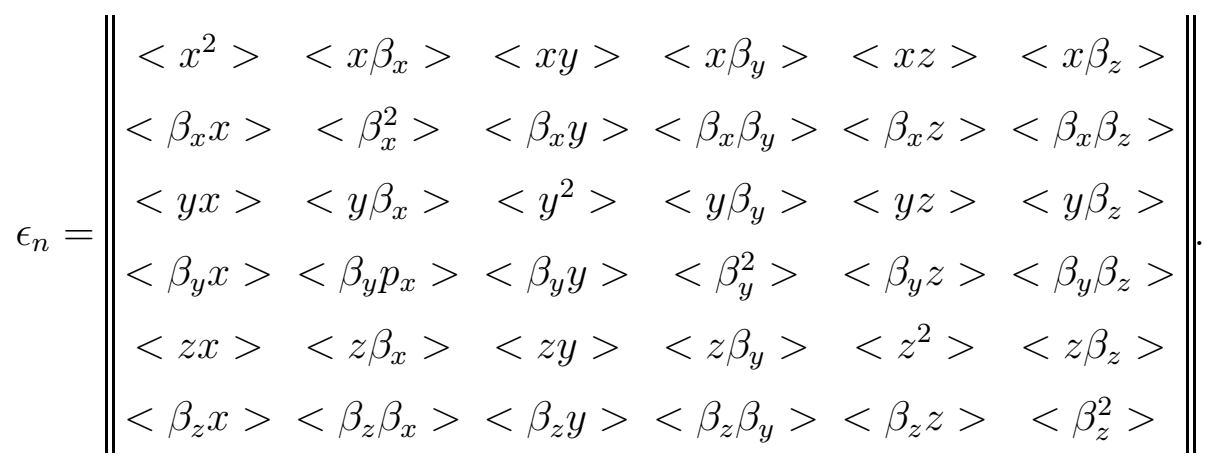

Before the main results of the simulation are shown, the specifics of the numerical tool and the choice of relevant parameters are described below. Since the electrons are nonrelativistic, the force among them is simply the Coulomb force. This allows us to use an existing tree-code package written by one of the original inventors of the technique [13]. The Coulomb force is calculated in the modified form of $\left(r^{2}+a^{2}\right)^{-2}$ (with $a=1.3 \mathrm{e}-12 \mathrm{~m}$ ) to avoid artificial collisions at short distance. The numerical integration is performed using the second-order symplectic integrator. The parameters that affect the accuracy are the (fixed) size of the time step $(\delta \mathrm{t})$ and the opening angle $(\theta)$ within which the electrons are lumped into one macro particle. Although this package has been tested extensively [13], we compared the transverse emittance over the time range of $12 \mathrm{ps}$ for different $\delta \mathrm{t}$ and $\theta$ under the initial conditions of our interest (see below). We find that convergence is reached for $\delta \mathrm{t}$ below 25 fs and $\theta$ below $1.5 \mathrm{rad}$. We chose $\delta \mathrm{t}$ of $12.4 \mathrm{fs}$ and $\theta$ of $0.8 \mathrm{rad}$ for our study.

The results of the simulation are presented below. To simplify the problem, all electrons are created at the same instant in time with the same energy and with zero transverse velocity. Furthermore, no external field is applied. As a result, the transverse emittance obtained here is the upper limit of that in an electron gun due to the absence of the longitudinal cooling and the thermalization between the transverse and the longitudinal phase 

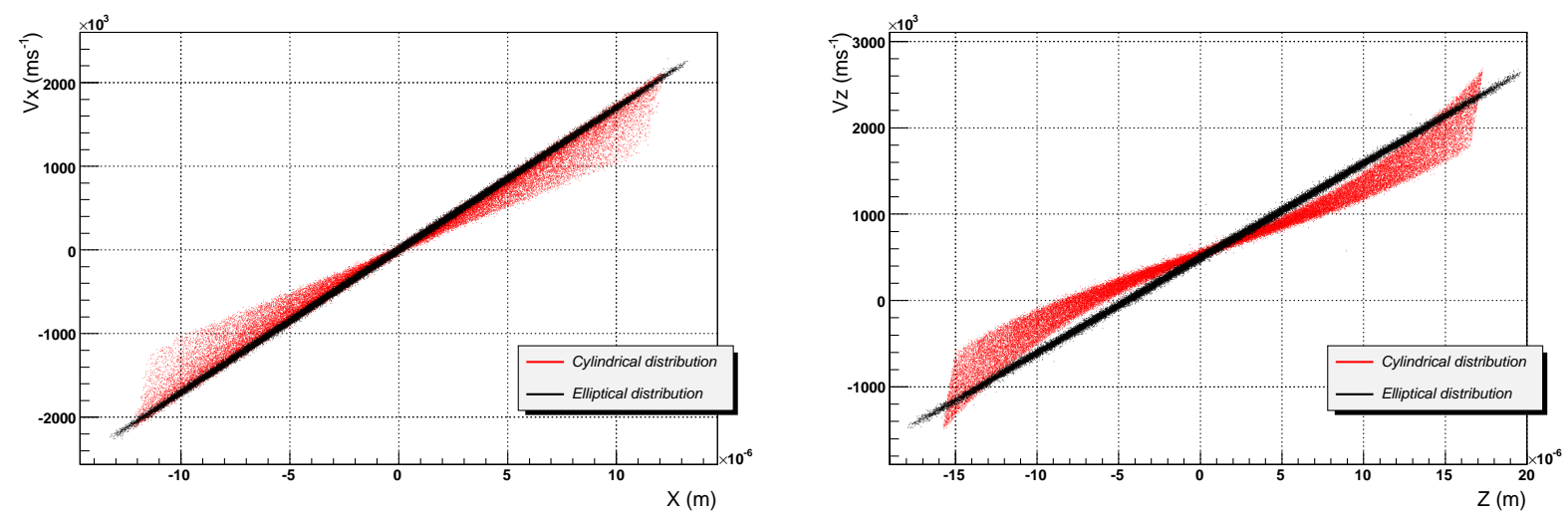

FIG. 1: Transverse (top) and longitudinal (bottom) phase space plots of the ellipsoidal and the cylindrical electron bunches at $t=1.5 \mathrm{ps}$. The number of electrons is $1048576\left(2^{20}\right)$ for both cases. The initial kinetic energy is $1 \mathrm{eV}$. For the cylindrical bunch, the radius is $10 \mu \mathrm{m}$ and the length is $30 \mu \mathrm{m}$. For the ellipsoidal bunch, the semi-axes are $10 \mu \mathrm{m}, 10 \mu \mathrm{m}$ and $15 \mu \mathrm{m}$.

spaces [14]. Figure 1 shows the transverse and longitudinal phase spaces of a cylindrical and an ellipsoidal bunches at $t=1.5$ ps. The linear nature of the latter shows that nonlinear correlations in the phase space are absent. In another word, ellipsoidal bunches remain ellipsoidal.

In order to test the validity of the code, the dependence of the $6 \mathrm{D}$ emittance on the spatial distributions of the electrons and the number of electrons has been studied (see Figure 2). The dependence of the $6 \mathrm{~d}$ emittance on the spatial distributions of the electrons is simulated using two cases of initial condition, both of which are spherical bunches. In the first case the electrons are distributed randomly throughout the spherical volume in uniform a distribution. In the second case the electrons are distributed on a $3 \mathrm{~d}$ cartesian grid within the same spherical volume, with constant grid spacing such that the number density and total number of electrons within the sphere is that of the first case. From the theoretical point of view, the $6 \mathrm{D}$ emittance of a spherical bunch with electrons launched from a Cartesian grid should be close to zero, with the residue coming from the finite size of the bunch and the edge effects. In our simulation, the tree algorithm, in principle, also contributes to the residual emittance as the tree structure will at some level disturb the symmetry of the distribution. Our results show that the value of the grid distribution is two to three orders of magnitude smaller than that for the random distribution. As shown 


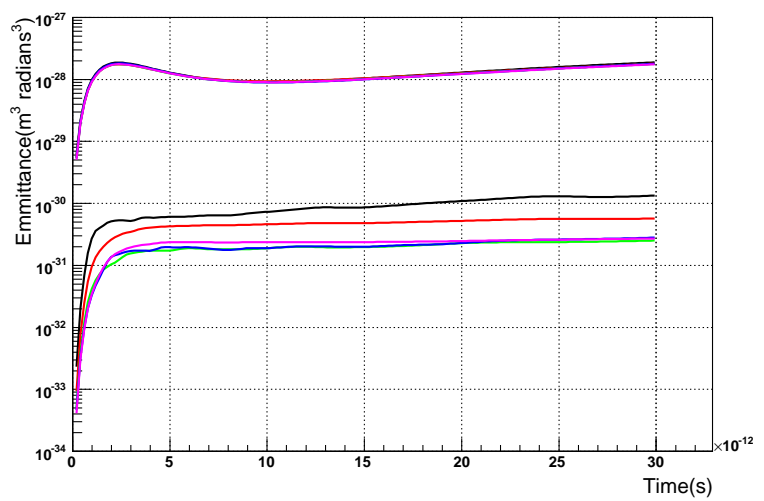

FIG. 2: 6D emittance plots of spherical electron bunches. All traces are normalized to the case with $2^{20}$ electrons. The longitudinal initial kinetic energy is $0.4 \mathrm{eV}$ for all cases. The number of electrons are $2^{17}, 2^{18}, 2^{19}, 2^{20}$ and $2^{21}$. The radius of each of the cases is $5 \mu \mathrm{m}, 6.300 \mu \mathrm{m}, 7.937$ $\mu \mathrm{m}, 10 \mu \mathrm{m}$ and $12.599 \mu \mathrm{m}$. The group of traces on the top are bunches with random spatial distributions and those on the bottom are bunches with electrons on the Cartesian grid (grid size $0.16 \mu \mathrm{m})$. For the bottom group, the traces for the cases of $2^{17}, 2^{18}, 2^{19}, 2^{20}$ and $2^{21}$ electrons are purple, red, green, blue and black, respectively. The average and the standard deviation at $\mathrm{t}=30$ ps for the random distribution are 1.803E-28 and 5.053E-30. Those for the grid case are 5.429E-31 and $4.637 \mathrm{E}-31$.

in Figure 2, the average emittance of the random distribution is 300 times larger than that of the grid distribution. The dependence of the emittance on the number of electrons is studied by varying the number of electron and the radius at the same time while keeping the number density constant. The fact that the standard deviation of the emittance for the grid distribution is $85 \%$ of the average entails that the emittance is not related to the number density. Hence we conclude that it is the result of the residual effects such as the finite size and edge of the sphere, the tree algorithm and numerical errors. On the other hand, the standard deviation for the random distribution is merely $3 \%$ of the average, showing that the emittance is due to the stochastic Coulomb interaction which is determined by the number density. It is clear that our code is working properly and the emitance is truely that generated by stochastic Coulomb interactions. Furthermore, this number is a good measure of the error bar of our results.

The relation between the transverse emittance and the number density is studied by 
varying the number of electrons while keeping the radius of the bunch fixed. Other initial conditions are the same as the cases discussed above. In figure 3, the transverse emittance as the function of time is plotted, which shows that the $6 \mathrm{D}$ emittance starts to saturate at $t$ $=100 \mathrm{fs}$ and increases with the number density. In order to study quantitatively the relation between the transverse emittance and the number density, the transverse emittance as the function of the number density is plotted (figure 4), together with an analytical estimation developed by Jansen [14, 15]. From Ref. [14, 15], which assumes that thermal equilibrium is reached, the kinetic energy per electron generated by potential energy relaxation is

$$
E_{k}=\frac{3}{2}\left(4 \pi a^{2}\right)^{1 / 3} C_{0} n^{1 / 3},
$$

where $n$ is the number density, $C_{0}=\frac{e^{2}}{4 \pi \epsilon_{0}}$ and $a=0.08702$. Assuming equal partition among the 3 degrees of freedom, the rms transverse emittance can be expressed as

$$
\epsilon_{x, y}=\frac{r}{2} \sqrt{\frac{2 E_{k}}{3 m_{e} c^{2}}}
$$

where $m_{e}$ is the mass of the electron and $c$ is the speed of light. As shown in figure 4, the numerical result is about 2 times larger than the analytical estimate. Furthermore, the scaling with number density is also different. In the analytical model, the emittance is proportional to one sixth power of the number density. Yet the empirical model fitted to the simulation result is

$$
\epsilon_{x, y}=C r n^{\alpha},
$$

where $C=2.62 \times 10^{-14}$ and $\alpha=0.319$. Note that the exponent almost doubles that of the analytical estimate. These differences may be due to the excess potential energy resulting from the fluctuations of the random distribution. Using eq. 5, we estimate that, for the case with $2^{20}$ electrons, around $40 \%$ of the excess potential energy, which is the difference between the random and grid distributions (52 meV out of the total amount of $90.6 \mathrm{eV}$ per electron), is transformed into uncorrelated kinetic energy. Furthermore, the lack of Debye screening may be another reason (see table I), since eq. 4 is obtained assuming Maxwell-Boltzmann distribution in the screened potential [14].

The simulation result and the empirical law help to answer the question that how "good" is a cathode good enough in terms of transverse emittance. For a given number density near the cathode, a cathode is good enough if the transverse emittance produced by the transverse momentum of the emitted electrons is as low as that generated by the Coulomb 


\begin{tabular}{|l|c|c|c|c|c|}
\hline$N$ & $2^{17}$ & $2^{18}$ & $2^{19}$ & $2^{20}$ & $2^{21}$ \\
\hline$n\left(10^{20} 1 / \mathrm{m}^{3}\right)$ & 0.313 & 0.626 & 1.252 & 2.503 & 5.007 \\
\hline$N_{D}$ & 0.072 & 0.101 & 0.135 & 0.186 & 0.254 \\
\hline$\omega_{p}(\mathrm{THz})$ & 0.316 & 0.446 & 0.631 & 0.893 & 1.262 \\
\hline
\end{tabular}

TABLE I: The radius is $10 \mu \mathrm{m}$; The quantities " $N$ ", " $n$ ", " $N_{D}$ " and " $\omega_{p}$ " are the number of electrons, the initial number density, the Debye number and the initial plasma frequency, respectively. Note that the Debye number is the estimate of the upper limit, which is obtained from eq. 5 by plugging in the final emittance obtained from simulation and the initial radius.

scattering. This serves to define a "stochastic limit" in terms of cathode performance. For a given current density $j$ and $r m s$ energy spread $E_{0}$, assuming homogeneous momentum distribution, the rms transverse and longitudinal velocities are

$$
v_{x}=v_{y}=v_{z}=\sqrt{\frac{2 E_{0}}{3 m_{e} c^{2}}} .
$$

Hence the number density near the cathode is

$$
n=\frac{j}{e \sqrt{\frac{2 E_{0}}{3 m_{e} c^{2}}}}
$$

and, from eq. 6, the transverse emittance due to potential energy relaxation is

$$
\epsilon_{x p}=C r\left(\frac{j}{e \sqrt{\frac{2 E_{0}}{3 m_{e} c^{2}}}}\right)^{\alpha} .
$$

Similarly to eq. 5, the transverse emittance due to the initial momentum distribution is

$$
\epsilon_{x k}=\frac{r}{2} \sqrt{\frac{2 E_{0}}{3 m_{e} c^{2}}} .
$$

and the optimum energy spread is then reached when $\epsilon_{x p}=\epsilon_{x k}$, which is

$$
E_{0}=\frac{3 m_{e} c^{2}}{2}\left[2 C\left(\frac{j}{e}\right)^{\alpha}\right]^{\frac{2}{\alpha+1}}
$$

As an example, let us consider the case of a bunch with a charge of $1 \mathrm{nC}$, pulse length of 10 ps and radius of $1 \mathrm{~mm}$. The peak current is $100 \mathrm{~A}$ and the peak current density is $3.2 \mathrm{e}+03$ $\mathrm{A} / \mathrm{cm}^{2}$. From eq. 11, the optimum energy spread is $29 \mathrm{meV}$. The minimum transverse emittance is the quadrature of eq. 9 and 10, which is $0.14 \mathrm{e}-6 \mathrm{~m}$ rad. Furthermore, when 


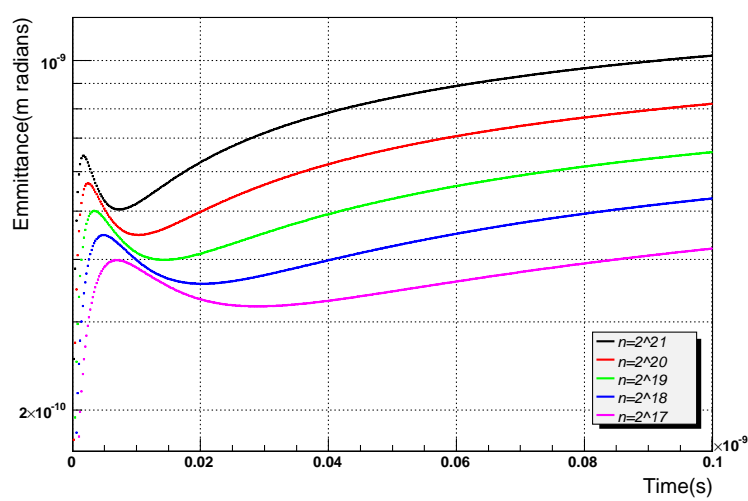

FIG. 3: Transverse emittance versus time of spherical electron bunches. The initial kinetic energy is $0.4 \mathrm{eV}$ and the radius is $10 \mu \mathrm{m}$ for all cases. The number of electrons are $2^{17}, 2^{18}, 2^{19}, 2^{20}$ and $2^{21}$.

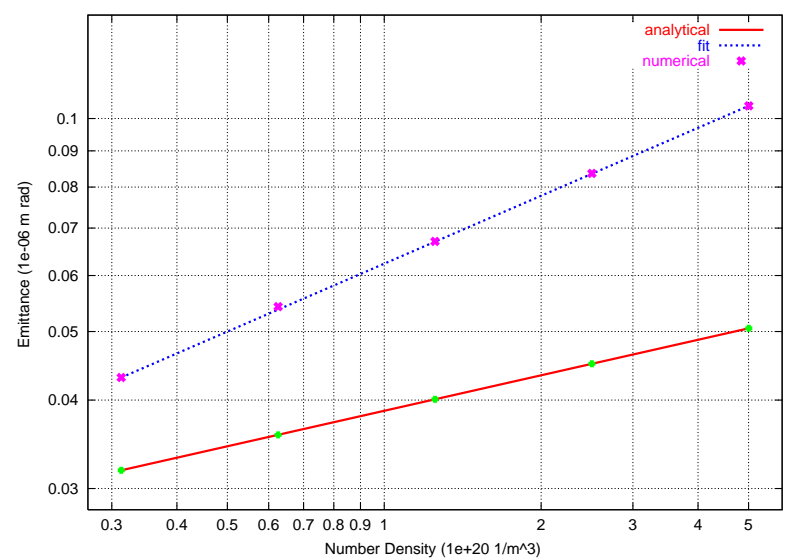

FIG. 4: Transverse emittance versus number density of spherical electron bunches. The initial kinetic energy is $0.4 \mathrm{eV}$ and the radius is $10 \mu \mathrm{m}$ for all cases. The number of electrons are $2^{17}, 2^{18}$, $2^{19}, 2^{20}$ and $2^{21}$. The emittance is scaled to the radius of $1 \mathrm{~mm}$. The function of the fitted curve is $\epsilon_{x}=2.62 \times 10^{-14} n^{0.319}$.

the average longitudinal energy $E_{a}$ is different from the energy spread $E_{0}$ and remains a constant, the optimum energy spread $E_{0} \propto j^{2 \alpha} / E_{a}^{\alpha}$. For larger $E_{a}$, the optimum energy spread is smaller and hence the minimum emittance.

In summary, we have shown through N-body calculation that there is a finite emittance associated with the stochastic Coulomb interaction among particles in an electron beam. 
This interaction was successfully reduced to a simple empirical law that should be of great value in accessing the conditions needed for achieving a defined emittance. Furtheremore, we have shown the benefit of launching electrons from a lattice, rather than randomly, something that may well be possible using field emitting tip arrays [16].

The authors gratefully acknowledges helpful conversations with S. Lidia, J. Corlett, M. Zolotorev, S. Zholents, B. Fawley, J. Qiang, F. Sannibale, D. Dowell, C. Limberg, I. Bazarov and Z. Huang. This work was supported by the U. S. Department of Energy under grant no. DE-AC02-05CH11231.

* christopher.coleman-smith@duke.edu

$\dagger$ WWan@lbl.gov

[1] T. Shintake, in Proceedings of the 2007 Particle Accelerator Conference, Albuquerque, NM (IEEE, Piscataway, NJ, 2007), pp. 89-93.

[2] H. Boersch, Zeitschr. Phys. 139, 115 (1954).

[3] G. H. Jansen, Coulomb Interactions in Particle Beams (Academic Press, New York, 1990), chap. 1.

[4] M. D. Nijkerk and P. Kruit, J. Appl. Phys. 96, 2985 (2004).

[5] S. J. Aarseth, Gravitational N-Body Simulations: Tools and Algorithms (Cambridge University Press, Cambirdge, 2003).

[6] J. M. Dawson, Rev. Mod. Phys. 55, 403 (1983).

[7] C. K. Birdsall and A. B. Langdon, Plasma Physics via Computer Simulation (McGraw-Hill, New York, 1985).

[8] J. Barnes and P. Hut, Nature 324, 446 (1986).

[9] B. E. Carlsten, Nucl. Instrum. Methods Phys. Res. A 285, 313 (1989).

[10] O. J. Luiten, Phys. Rev. Lett. 93, 094802 (2004).

[11] E. J. Routh, A Treatise on Analytical Statics (Cambridge University Press, Cambridge, 1892), vol. 2, pp. 101-109.

[12] V. Danilov, Phys. Rev. ST Accel. Beams 6, 094202 (2003).

[13] J. E. Barnes, J. Comp. Phys. 87, 161 (1990).

[14] G. H. Jansen, Coulomb Interactions in Particle Beams (Academic Press, New York, 1990), 
chap. 3, pp. 55-57.

[15] J. Orloff, ed., Handbook of Charged Particle Optics (CRC Press, New York, 1997), chap. 7, p. 306.

[16] K. B. K. Teo, Nature 437, 968 (2005). 